\title{
Detection of Serum Biochemical Markers in Elderly Patients with Type 2 Diabetes Mellitus and Its Relationship with Coronary Heart Disease
}

\author{
Junran Zhang ${ }^{1,}{ }^{*}$, Kaiyan Shao ${ }^{2}$, Shuguo Yang ${ }^{1}$ and Qinghua $\mathrm{Ma}^{1}$ \\ ${ }^{1}$ Department of Cardiology, Linyi Central Hospital, Linyi, China \\ 2 Intensive Care Unit, Linyi Central Hospital, Linyi, China \\ * Corresponding author: Junran Zhang, Department of Cardiology, Linyi Central Hospital, Linyi, China. Tel: +86037155966339; Email: junranzhang@sina.com
}

Received 2020 June 27; Revised 2020 July 11; Accepted 2020 July 29.

\begin{abstract}
Background: Coronary heart disease (CHD) is myocardial ischemia caused by coronary atherosclerosis and stenosis.

Objectives: This study aimed to investigate the relationship of CHD with glycated albumin (GA), glycosylated hemoglobin (HbA 1c), glucose (Glu), homocysteine (Hcy), triglyceride (TG), and apolipop protein A (apoA) levels in elderly patients with type 2 diabetes mellitus (T2DM). Moreover, it was attempted to predict which changes had a significant correlation with the occurrence of CHD in these biochemical indicators.

Methods: This clinical cohort study included a total of 472 patients admitted to our hospital from June 2018 to June 2019. They were then divided into three groups of concurrent ( $\mathrm{n}=168$, T2DM complicated with CHD), DM ( $\mathrm{n}=148$, T2DM alone), and control ( $\mathrm{n}=156$, healthy individuals).

Results: There were significant differences between the disease group (i.e., DM and concurrent groups) and control group in terms of GA $(\mathrm{P}=0.013,28.58 \pm 8.01$ vs. $15.77 \pm 1.44)$, $\mathrm{HbA} 1 \mathrm{c}(\mathrm{P}=0.022,9.5 \pm 1.5$ vs. $5.5 \pm 0.5)$, Glu $(\mathrm{P}=0.012,8.54 \pm 2.23$ vs. $4.12 \pm 0.39), \mathrm{Hcy}(\mathrm{P}=0.031$, $11.16 \pm 3.28$ vs. $5.03 \pm 2.87)$, $T G(P=0.021,1.83 \pm 0.49$ vs. $0.84 \pm 0.18)$, and apoA $(\mathrm{P}=0.031,1.10 \pm 0.12$ vs. $1.30 \pm 0.18)$.

Moreover, GA ( $\mathrm{P}=0.025,27.14 \pm 6.34$ vs. $28.58 \pm 8.01$; concurrent group vs. $\mathrm{DM}$ group), $\mathrm{HbA} 1 \mathrm{c}(\mathrm{P}=0.033,8.3 \pm 1.2$ vs. 9.5 \pm 1.5 ; concurrent group vs. DM group), Glu ( $\mathrm{P}=0.019,8.62 \pm 3.56$ vs. $8.54 \pm 2.23$; concurrent group vs. DM group), Hcy ( $\mathrm{P}=0.031,17.56 \pm 6.36$ vs. 11.16 \pm 3.28 ; concurrent group vs. DM group), total cholesterol ([TC); $\mathrm{P}=0.022,3.06 \pm 0.20$ vs. $3.69 \pm 0.29$; concurrent group vs. DM group), and low-density lipoprotein ([LDL); $P=0.037,2.57 \pm 1.02$ vs. $3.40 \pm 1.17$; concurrent group vs. DM group) in disease group (DM group and concurrent group) were higher than those in the control group; however, apoA $(\mathrm{P}=0.023,0.95 \pm 0.12$ vs. $1.10 \pm 0.12$; concurrent group vs. DM group) in disease group was lower than that in the control group. Furthermore, there were significant differences between the disease and control groups in terms of GA, HbA 1c, Glu, Hcy, TC, LDL, and apoA ( $\mathrm{P}<0.05)$. Pearson correlation analysis between DM and concurrent groups was performed on the clinical parameters with statistical differences, and GA was highly correlated with $\mathrm{HbA} 1 \mathrm{c}$ and Glu $(\mathrm{P}<0.01)$.

Conclusion: High-risk patient screening with high levels of GA, Hcy, and apoA in elderly patients with T2DM can not only improve the symptoms of patients with targeted treatment but also reduce the incidence of CHD by timely intervention, which is of great significance to improve the quality of life of patients.
\end{abstract}

Keywords: Apolipop protein A, Coronary heart disease, Glycated albumin, Homocysteine, Type 2 diabetes mellitus

\section{Background}

Coronary heart disease (CHD) is myocardial ischemia caused by coronary atherosclerosis and stenosis. It is a cardiovascular disease that seriously threatens the health of human life (1). Its incidence has increased year by year and is developing in younger adults. About $70 \%$ of patients with CHD have abnormal blood glucose. Type 2 diabetes mellitus (T2DM) is considered an independent risk factor for cardiovascular disease. In patients with T2DM, coronary atherosclerotic heart disease has an early onset, high disease rate, and severe coronary lesions. The risk of stroke and cardiovascular death in the T2DM population is nearly 4 times higher than that in the non-T2DM population. In addition to the high-risk factors (i.e., dyslipidemia, hypertension, smoking, age, obesity, hyperhomocysteinemia) of the disease, T2DM is also a risk factor for the development of CHD (2). The incidence of T2DM patients complicated with CHD can be as high as $72.3 \%$, which is 2 to 4 times that of non-diabetic patients (3). Long-term effective blood glucose monitoring has an important clinical guiding value for reducing T2DM complications (4). According to the Chinese Guidelines for Clinical Application of Blood Glucose Monitoring, the determination of glycated albumin (GA) has become one of the effective methods for blood glucose monitoring in patients with T2DM, and its evaluation of diabetic vascular complications has also important clinical value (5). However, there is little data on controlling blood glucose in elderly patients with T2DM. Furthermore, there is no conclusion about the GA risk factors associated with T2DM in China (6).

\section{Objectives}

This study aimed to investigate the relationship of CHD with GA, glycosylated hemoglobin (HbA 1c), glucose (Glu), homocysteine (Hcy), triglyceride (TG), and apolipop protein A (apoA) levels in elderly patients with T2DM. Moreover, it was attempted to predict which changes in these biochemical 
indicators had a significant correlation with the occurrence of CHD.

\section{Methods}

This clinical cohort study included a total of 472 patients admitted to our hospital from June 2018 to June 2019. They were then divided into three groups of concurrent ( $n=168$, T2DM complicated with CHD), DM ( $n=148$, T2DM alone), and control ( $n=156$, healthy individuals). Concurrent and DM groups collectively referred to as the disease group, and patients in the control group were selected from the physical examination center of our hospital.

Subsequently, the blood samples of the patients were taken on an empty stomach, and the serum was taken after centrifugation. The inclusion criteria were: 1) fulfillment of the diagnostic criteria for T2DM in the 2007 edition of Chinese Guidelines for Diabetes Prevention and Control, 2) normal cognition and clear mind, and 3) lack of serious external trauma recently. It should be mentioned that the patients in the control group met the diagnostic criteria for CHD after clinical diagnosis.

On the other hand, the patients who were allergic to the test equipment and drugs; those with hearing, comprehension, vision, and reading disorders; the cases who exited the research procedure during diagnosis and treatment; and the individuals with the combination of the severe immune blood system, nervous system or malignant tumor were excluded from the study.

The study protocol was approved by the Ethics Committee of Linyi Central Hospital, Shandong, China. It is worth mentioning that informed consent was obtained from the patients.

The control group included 156 healthy cases (78 males and 78 females) who referred to our Health Checkup Center for physical examinations with a mean age of $76.65 \pm 5.50$ years (age range: 70-81 years). Physical and internal surgery routine examinations were required, and the inclusion criteria were lack of malignant tumors, chronic infectious diseases, and organ dysfunction, as well as normality of blood routine, liver function, kidney function, blood lipids, and blood glucose indicators.

The DM group included 168 T2DM patients without CHD (84 males and 84 females) and with the mean age of $79.23 \pm 7.42$ years (age range: $75-83$ years). The inclusion criteria were: 1) fulfillment of the World Health Organization diagnostic criteria for diabetes: $\mathrm{HbA} 1 \mathrm{c} \geq 6.5 \%$, or fasting plasma glucose (FPG) $\geq 7.0 \mathrm{mmol} / \mathrm{L}$ (fasting is defined as no calorie intake for at least $8 \mathrm{~h}$ ), 2) oral glucose tolerance test $2 \mathrm{~h}$ blood glucose $\geq 11.1 \mathrm{mmol} / \mathrm{L}$, and 3) typical hyperglycemia or hyperglycemia crisis and random blood glucose $\geq 11.1 \mathrm{mmol} / \mathrm{L}$. On the other hand, patients with malignant tumors, chronic infectious diseases, organ dysfunction, and immune dysfunction were excluded from the study.

The concurrent group included 148 T2DM patients with CHD (74 males and 74 females) and a mean age of $76.33 \pm 6.23$ years (age range: $72-83$ years). In addition to typical clinical symptoms, subjects were also required evidence of myocardial ischemia or coronary obstruction, as well as markers of myocardial injury. Related examination methods included conventional electrocardiogram (ECG) and electrocardiogram load test, radionuclide myocardial imaging, coronary angiography, and intravascular ultrasound.

Dynamic ECG diagnostic criteria were ST-segment drops more than $0.05 \mathrm{mV}$ and ST-segment increase above $0.2 \mathrm{mV}$ for a duration of no less than $2 \mathrm{~min}$. The diagnostic criterion for CHD is the coronary artery stenosis more than $50 \%$ after coronary angiography. It is worth mentioning that patients with malignant tumors or chronic infectious diseases, organ failure, and immune dysfunction were excluded from the study. In this study, the overall sample statistics followed a normal distribution, and the samples were screened according to the normal distribution of the sample statistics.

The Glu, TG, total cholesterol (TC), low-density lipoprotein (LDL), high-density lipoprotein (HDL), apoA, and apolipop protein B (apoB) were determined by Cobas8000 automatic biochemical analyzer (Roche Company, Switzerland) and its supporting reagents. Furthermore, the Glu was tested by hexose kinase method, and TC as well as TG were treated by cholesterol oxidase (Zhejiang Ruixin Pharmaceutical Co., Ltd., China) and oxidase (Shandong Hualu Pharmaceutical Co., Ltd., China), respectively. The LDL and HDL adopt homogeneous enzyme colorimetry; moreover, the apoA and apoB adopted the immunoturbidimetric method. The DXC800 automatic biochemical analyzer (Beckman, USA) and its supporting reagents were used to detect GA (using solid-state liquid enzymatic method) and Hcy (using the cyclic enzymatic method). The HbA 1c was measured by the HA-8180 fully automatic glycated hemoglobin analyzer (Aikelai, Japanese) and its supporting reagents. In addition, it was detected by the micro-column method. The original quality control serum was selected using quality control products, and the quality control products before the sample measured every day were tested to ensure that the items tested by the indoor quality control were under control.

\subsection{Statistical analysis}

The data were analyzed in SPSS software (version 17.0), and the results were expressed as mean \pm SD $(\mathrm{x} \pm \mathrm{s})$. Moreover, one-way analysis of variance (ANOVA) was used to make a comparison among the three groups. Additionally, Pearson correlation analysis between DM and concurrent groups was performed on clinical parameters with statistical 
differences, and the correlation between parameters was also investigated in this study. Dual regression analysis between DM and concurrent groups was performed at the same time. Continuous variables were tested for normality, and one-way ANOVA was used to make multiple comparisons among the groups. In this study, the normal assumptions were made, and the non-parametric method was used for the variables that failed to meet the normal distribution. A p-value less than 0.05 was considered statistically significant.

\section{Results}

This study included 472 patients admitted to our hospital from June 2018 to June 2019. They were then divided into three groups of concurrent $(n=168, T 2 D M$ complicated with CHD, DM ( $n=148$, T2DM alone), and control ( $n=156$, healthy controls). The concurrent and DM groups collectively referred to as the disease group, and the patients in the control group were selected from those who referred for a physical examination to our center in the hospital. There were no significant differences among the groups in terms of the demographic characteristics, such as height, weight, body mass index, as well as systolic and diastolic blood pressure $(\mathrm{P}>0.05)$.

Furthermore, no significant differences were observed between the disease and control groups regarding GA, $\mathrm{HbA} 1 \mathrm{c}, \mathrm{Glu}, \mathrm{Hcy}, \mathrm{TG}$, and apoA $(\mathrm{P}<0.05)$. The GA $(\mathrm{P}=0.013)$, HbA 1c $(\mathrm{P}=0.022)$, Glu $(\mathrm{P}=0.012)$, Hcy $(\mathrm{P}=0.031)$, and $\mathrm{TG}(\mathrm{P}=0.021)$ were higher in the disease group, compared to the control group. However, the apoA $(\mathrm{P}=0.031)$ in the disease group was lower than that in the control group.

According to the results, there were significant differences between the concurrent and DM groups $(\mathrm{P}<0.05)$ in terms of GA $(\mathrm{P}=0.025), \mathrm{HbA} 1 \mathrm{c}(\mathrm{P}=0.033)$, Glu $(\mathrm{P}=0.019)$, Hcy $(\mathrm{P}=0.031)$, TC $(\mathrm{P}=0.022)$, LDL $(\mathrm{P}=0.037)$, and apoA $(\mathrm{P}=0.023)$. Table 1 tabulates the differences among the three groups in terms of GA, HbA 1c, Glu, Hcy, and apoA.

There was a significant difference between DM and concurrent groups in terms of $\mathrm{HbA} 1 \mathrm{c}$ $(\mathrm{P}=0.007)$, Glu $(\mathrm{P}=0.004)$, and GA $(\mathrm{P}=0.009)$. However, no significant difference was observed between these two groups regarding Hcy, apoA, and GA $(\mathrm{P}>0.05)$.

The correlation coefficients between HbA $1 \mathrm{c}$ and GA as well as Glu and GA were 0.680, and 0.465, respectively. It should be noted that the correlation between $\mathrm{HbA} 1 \mathrm{c}$ and GA was more significant than that between Glu and GA (Table 2). The 95\% confidence intervals of GA, Hcy, and apoA were 0.765 , $0.962 ; 1.015,1.201$; and $0.004,0.403$, respectively. A p-value of $\mathrm{HbA} 1 \mathrm{c}$ and Glu was over 0.05 which showed no significant differences (Table 3 ).

\begin{tabular}{lccc}
\hline \multicolumn{4}{l}{ Table 1. Comparison between the disease and control groups in terms of clinical parameters } \\
\hline Items & Control group (n=156) & DM group (n=168) & Concurrent group (n=148) \\
\hline GA (\%) & $15.77 \pm 1.44$ & $28.58 \pm 8.01^{\mathrm{a}}$ & $27.14 \pm 6.34^{\mathrm{ab}}$ \\
HbA 1C (\%) & $5.5 \pm 0.5$ & $9.5 \pm 1.5^{\mathrm{a}}$ & $8.3 \pm 1.2^{\mathrm{ab}}$ \\
Glu (mmol/L) & $4.12 \pm 0.39$ & $8.54 \pm 2.23^{\mathrm{a}}$ & $8.62 \pm 3.56^{\mathrm{ab}}$ \\
Hcy (mmol/L) & $5.03 \pm 2.87$ & $11.16 \pm 3.28^{\mathrm{a}}$ & $17.56 \pm 6.36^{\mathrm{a}}$ \\
TG (mmol/L) & $0.84 \pm 0.18$ & $1.83 \pm 0.49^{\mathrm{a}}$ & $1.25 \pm 0.61^{\mathrm{a}}$ \\
TC (mmol/L) & $3.23 \pm 0.66$ & $3.69 \pm 0.29$ & $3.06 \pm 0.20^{\mathrm{b}}$ \\
HDL (mmol/L) & $1.35 \pm 0.15$ & $1.01 \pm 0.30^{\mathrm{a}}$ & $1.11 \pm 0.28^{\mathrm{a}}$ \\
LDL (mmol/L) & $2.58 \pm 0.45$ & $3.40 \pm 1.17^{\mathrm{a}}$ & $2.57 \pm 1.02^{\mathrm{b}}$ \\
apoA (g/L) & $1.30 \pm 0.18$ & $1.10 \pm 0.12^{\mathrm{a}}$ & $0.95 \pm 0.12^{\mathrm{ab}}$ \\
apoB (g/L) & $0.91 \pm 0.51$ & $1.01 \pm 0.32$ & $1.03 \pm 1.02$ \\
\hline
\end{tabular}

Note: GA: glycated albumin; HbA 1c: glycosylated hemoglobin; Glu: glucose; Hcy: homocysteine; TG: triglyceride; TC: total cholesterol; HDL: high-density lipoprotein; LDL: low-density lipoprotein. Compared with the control group, aP $<0.05$; compared with the $\mathrm{DM}$ group, bP $<0.05$.

Table 2. Correlation analysis between DM and concurrent groups in terms of GA and other clinical parameters

\begin{tabular}{lcccc}
\hline Items & HbA 1c & Glu & Hcy & \\
\hline $\boldsymbol{r}$ & $0.680^{* *}$ & $0.485^{* *}$ & 0.023 & 0.014 \\
$\mathbf{P}$ & 0.001 & 0.000 & 0.642 & 0.703 \\
\hline
\end{tabular}

Note: GA: glycated albumin; HbA 1c: glycosylated hemoglobin; Glu: glucose; Hcy: homocysteine. ${ }^{* *} \mathrm{P}<0.01$; ${ }^{*} \mathrm{P}<0.05, r$ is the correlation coefficient.

\begin{tabular}{|c|c|c|c|c|c|}
\hline Items & $\mathbf{b}$ & Standard error & $\mathbf{P}$ & OR & Confidence interval \\
\hline GA & -0.123 & 0.048 & $0.009^{* *}$ & 0.846 & $0.765-0.962$ \\
\hline HbA 1c & -0.098 & 0.171 & 0.562 & 0.925 & $0.648-1.265$ \\
\hline Glu & -0.063 & 0.006 & 0.539 & 0.948 & $0.799-1.126$ \\
\hline Hсy & -0.079 & 0.043 & $0.021^{*}$ & 1.156 & $1.015-1.201$ \\
\hline apoA & -3.140 & 1.189 & $0.006^{* *}$ & 0.039 & $0.004-0.406$ \\
\hline
\end{tabular}

Note: GA: glycated albumin; HbA 1c: glycosylated hemoglobin; Glu: glucose; Hcy: homocysteine. ${ }^{* *} \mathrm{P}<0.01$; * $\mathrm{P}<0.05$; "b" is the partial regression coefficient; OR is the odds ratio. 


\section{Discussion}

The GA is mainly used to monitor the mean blood glucose level in the past 2-4 weeks in T2DM patients (7). According to a study, it was found that GA $\geq 17.1 \%$ could screen out most undiagnosed diabetic patients (8). In addition to being able to monitor the patient's blood glucose status, GA is more advantageous in determining the speed of treatment and adjusting the timeliness of administration (9). At the same time, for patients with reduced $\mathrm{HbA} 1 \mathrm{c}$ detection accuracy due to hemoglobin variation, GA can also be used as a necessary supplement that is not affected by red blood cell changes (10). As an early glycosylation product, GA is transformed and accumulated in the blood vessels causing vascular damage (especially to the microvessels) and leading to vascular sclerosis, stenosis, and obstruction, thereby promoting the occurrence of diabetic microvascular complications $(11,12)$.

Diabetes as one of the high-risk risks of CHD is one of the risk factors for T2DM patients complicated with CHD (13). This is an early and effective warning of high-risk CHD in patients with diabetes and also a timely screening of high-risk patients. It is especially important to reduce the incidence of adverse cardiovascular events and mortality (14). According to the Chinese Guidelines for the Prevention and Treatment of Type 2 Diabetes, most patients with T2DM have metabolic syndrome, which is accompanied by hypertension, hyperglycemia, and dyslipidemia $(15,16)$.

In this study, GA, HbA 1c, Glu, Hcy, and TG in the disease group were higher than those in the control group; however, the disease group had lower levels of HDL and apoA, compared to the control group, indicating that the results of the disease group were consistent with the Chinese Guidelines for the Prevention and Treatment of Type 2 Diabetes. There were significant differences between DM and concurrent groups in terms of GA, HbA 1c, Glu, Hcy, TC, LDL, and apoA $(\mathrm{P}<0.05)$. Moreover, the three groups were significantly different regarding $\mathrm{GA}, \mathrm{HbA}$ 1c, Glu, Hcy, and apoA. Individual lipid profiles, including LDL, TC, and apoA abnormalities play an important role in the formation of coronary atherosclerosis; in addition, they have important clinical significance in the diagnosis of CAD, which is consistent with the results of the literature and fully confirmed the representativeness of the relevant clinical parameters included in the disease group (17). However, GA, HbA 1c, and Glu are the most representative indicators of blood glucose control in T2DM patients (18). The HbA 1c is the international gold standard for monitoring long-term glycemic control and is an indicator of glycemic control status in the past 1-2 months (19). A prospective study of diabetes in the United Kingdom found that for every $1 \%$ increase in $\mathrm{HbA} 1 \mathrm{c}$, the risk of cardiovascular disease increased by $1 \%(20)$. The Glu value is the diagnostic cut-off point of T2DM. It is easy to measure at the bedside and grasp the static instantaneous blood glucose level of the patient; however, it is very susceptible to various factors (21). The $\mathrm{HbA} 1 \mathrm{c}$ and Glu play an important role in the development of CHD (3). Table 2 summarizes the correlation of GA with HbA $1 \mathrm{c}$ and Glu.

In the development of CHD, the changes of HbA $1 \mathrm{c}$ and Glu will inevitably lead to the change of GA; accordingly, GA has a certain effect on the progress of CHD. However, it is found in Table 3 that HbA 1c and Glu are highly correlated with GA in the concurrent group, which has no significance in the regression analysis $(\mathrm{P}>0.05)$ suggesting that $\mathrm{HbA} 1 \mathrm{c}$ and Glu can promote the development of CHD and have differences based on diabetes. Nonetheless, it cannot be a risk factor for T2DM complicated with CHD. However, GA showed a significant correlation in this regard $(\mathrm{P}<0.01)$. It can be seen that $\mathrm{GA}$ level monitoring is more predictive than $\mathrm{HbA} 1 \mathrm{c}$ and Glu for patients with potential CHD complicated with T2DM.

In addition to abnormally elevated blood glucose in patients with T2DM, this study also showed that T2DM was associated with high Hcy and low apoA. The Hcy is a vascular injury-causing amino acid, and hyperhomocysteinemia is associated with vascular endothelial cell injury and atherosclerosis (22). The apoA is an apolipoprotein closely related to HDL. According to the Framingham Heart Study, the risk of CHD decreased by $2 \%$ to $3 \%$ for every $0.03 \mathrm{mmol} / \mathrm{L}$ increase in HDL (23). As a protective factor, apoA reduces the corresponding protective effect of T2DM and accelerates the development of CHD (24). This study verified the above literature using the results of regression analysis. Table 3 displays that Hcy and apoA in patients with T2DM have statistical significance for complicated CHD $(\mathrm{P}<0.05)$. The apoA is reduced, and the trend of T2DM complicated with CHD is revealed with an increase in Hcy.

For diabetic patients, cardiovascular complications must be also prevented in addition to control blood glucose. Therefore, it is of great significance to search for early cardiovascular disease monitoring indicators of T2DM to guide the direction of clinical treatment. This study mainly discussed the relevance of GA to T2DM complicated with CHD. There is a significant correlation between T2DM complicated with CHD. Therefore, in the clinical stage, GA levels are highly correlated with $\mathrm{HbA} 1 \mathrm{c}$ and Glu that should be monitored early in T2DM, and potential CHD patients should be screened for early treatment. In addition, this study found that apoA was a protective factor for CHD complicated with T2DM, and Hcy was a risk factor. Combined with changes in Hcy and apoA, it was helpful for clinicians to understand the development and outcome of T2DM patients and improve the accuracy of screening. This study 
discussed the detection of serum biochemical indicators in elderly patients with T2DM and its relationship with CHD for the first time. Although previous studies have found a correlation between T2DM and the occurrence of CHD, there is no clear discussion from the perspective of blood biochemical indicators, and it cannot be used in clinical practice.

This study explored the relevance of CHD from the perspective of clinical biochemical indicators and guided the diagnosis, treatment, and prognosis of clinical diseases from a certain level. Moreover, this study set the research object to the elderly, a highrisk group of CHD and diabetes, which had more clinical significance.

\section{Conclusion}

High-level monitoring of the of GA, Hcy, and apoA in elderly patients with T2DM can not only improve the symptoms of patients with targeted treatment but also reduce the incidence of $\mathrm{CHD}$ by timely intervention, which is of great significance to improve the quality of life of high-risk patients. The GA, Hcy, and apoA may be important predictors of CHD in elderly diabetic patients. This study first proposed the indicators of cardiovascular disease in elderly diabetic patients, which were easily available in clinical testing.

Regarding the limitations of this study, one can name the focus on clinical patient data from one of our hospitals and a lack of multi-center research data. Due to the limited number of cases collected, it is still not enough to discuss whether GA is a factor in CHD complicated with T2DM. It is necessary to collect a large number of cases later for further discussion. The future investigations are recommended to focus on GA's trend research on T2DM complicated with CHD, and conduct a large number of prospective experiments on the subject selection group.

\section{Acknowledgements}

None to declare.

\section{References}

1. Giannopoulos S, Texakalidis P, Charisis N, Jonnalagadda AK, Chaitidis N, Giannopoulos S, et al. Synchronous carotid endarterectomy and coronary artery bypass graft (CABG) versus staged carotid artery stenting and CABG for patients with concomitant severe coronary and carotid stenosis: a systematic review and meta-analysis. Ann Vasc Surg. 2020;62:463-73. doi: 10.1016/j.avsg.2019.06.018. [PubMed: 31449948].

2. Harky A, Noshirwani A, Karadakhy 0, Ang J. Comprehensive literature review of anomalies of the coronary arteries. J Card Surg. 2019;34(11):1328-43. doi: 10.1111/jocs.14228. [PubMed: 31449703].

3. Logan N, Islam MS, Chughtai JZ, Murphy NF. An atypical cause of myocardial infarction: case report of an obstructing papillary fibroelastoma of the aortic valve. Eur Heart J Case Rep. 2019;3(2):ytz058. doi: 10.1093/ehjcr/ytz058. [PubMed:
31449619].

4. Mortada I. Hyperuricemia, type 2 diabetes mellitus, and hypertension: an emerging association. Curr Hypertens Rep. 2017;19(9):69. doi: 10.1007/s11906-017-0770-x. [PubMed: 28770533].

5. Gao Y, Peterson E, Pagidipati N. Opportunities for improving use of evidence-based therapy in patients with type 2 diabetes and cardiovascular disease. Clin Cardiol. 2019;42(11):1063-70. doi: 10.1002/clc.23252. [PubMed: 31448852].

6. Ramachandran S, Hackett GI, Strange RC. Sex hormone binding globulin: a review of its interactions with testosterone and age, and its impact on mortality in men with type 2 diabetes. Sex Med Rev. 2019;7(4):669-78. doi: 10.1016/j.sxmr.2019.06.006. [PubMed: 31447413].

7. Naito R, Miyauchi K. Coronary artery disease and type 2 diabetes mellitus. Int Heart J. 2017;58(4):475-80. doi: 10.1536/ihj.17-191. [PubMed: 28717115].

8. Xu H, Sun L, Miao C, Jin Y, Hou Y. Type 2 diabetes mellitus is associated with increased left ventricular mass independent of coronary artery volume. Clin Radiol. 2019;74(12):972.e17-23. doi: 10.1016/j.crad.2019.07.010. [PubMed: 31420187].

9. Tahara N, Nitta Y, Bekki M, Tahara A, Maeda-Ogata S, Sugiyama $\mathrm{Y}$, et al. Two-hour postload plasma glucose and pigment epithelium-derived factor levels are markers of coronary artery inflammation in type 2 diabetic patients. J Nucl Cardiol. 2019;27(4):1352-64. doi: 10.1007/s12350-019-01842-5. [PubMed: 31407236].

10. Xiong SL, Lim GT, Yin SJ, Lee J, Lee JR, Hahn MJ, et al. Inhibitory effect of $\alpha$-ketoglutaric acid on $\alpha$-glucosidase: integrating molecular dynamics simulation and inhibition kinetics. $J$ Biomol Struct Dyn. 2019;38(12):3496-503. doi: 10.1080/ 07391102.2019.1659858. [PubMed: 31448679].

11. Seah JY, Ong CN, Koh WP, Yuan JM, van Dam RM. A dietary pattern derived from reduced rank regression and fatty acid biomarkers is associated with lower risk of type 2 diabetes and coronary artery disease in Chinese adults. J Nutr. 2019;149(11):2001-10. doi: 10.1093/jn/nxz164. [PubMed: 31386157].

12. Mráz M, Cinkajzlová A, Kloučková J, Lacinová Z, Kratochvílová H, Lipš M, et al. Dendritic cells in subcutaneous and epicardial adipose tissue of subjects with type 2 diabetes, obesity, and coronary artery disease. Mediators Inflamm. 2019;2019: 5481725. doi: 10.1155/2019/5481725. [PubMed: 31210749].

13. Tsantes AE, Taichert M, Kyriakou E, Katogiannis $K$, Lytras T, Gialeraki A, et al. The prognostic value of multiple electrode aggregometry and light transmittance aggregometry in stable cardiovascular patients with type 2 diabetes mellitus. Thromb Res. 2019;180:47-54. doi: 10.1016/j.thromres.2019.06.001. [PubMed: 31202191].

14. Emamalipour M, Seidi K, Jahanban-Esfahlan A, JahanbanEsfahlan R. Implications of resistin in type 2 diabetes mellitus and coronary artery disease: Impairing insulin function and inducing pro-inflammatory cytokines. J Cell Physiol. 2019; 234(12):21758-69. doi: 10.1002/jcp.28913. [PubMed: 31187484].

15. Anholm C, Kumarathurai P, Jürs A, Pedersen LR, Nielsen OW, Kristiansen OP, et al. Liraglutide improves the beta-cell function without increasing insulin secretion during a mixed meal in patients, who exhibit well-controlled type 2 diabetes and coronary artery disease. Diabetol Metab Syndr. 2019;11:42. doi: 10.1186/s13098-019-0438-6. [PubMed: 31164926].

16. Zhou JB, Zhu XR, Zhao W, Yin L, Li HB, Qi L, et al. Prediction of proliferative diabetic retinopathy to asymptomatic obstructive coronary artery disease in chinese type 2 diabetes individuals: an exploratory study. Metab Syndr Relat Disord. 2019;17(7):367-73. doi: 10.1089/met.2018.0140. [PubMed: 31145036].

17. Azzam SK, Osman WM, Lee S, Khalaf $\mathrm{K}$, Khandoker $\mathrm{AH}$, Almahmeed W, et al. Genetic associations with diabetic retinopathy and coronary artery disease in Emirati patients with type-2 diabetes mellitus. Front Endocrinol (Lausanne). 2019;10:283. doi: 10.3389/fendo.2019.00283. [PubMed: 31130920]. 
18. Senyigit A, Uzun H, Gultepe I, Konukoglu D. The relationship between carotid intima-media thickness and serum secreted frizzled-related protein-4 and dipeptidyl peptidase-4 in diabetic patients with cardiovascular diseases. Bratisl Lek Listy. 2019;120(3):188-94. doi: 10.4149/BLL_2019_032. [PubMed: 31023036].

19. Eakin KA, Saleem M, Herrmann N, Cogo-Moreira H, Mielke MM, Oh PI, et al. Plasma sphingolipids mediate a relationship between type 2 diabetes and memory outcomes in patients with coronary artery disease undertaking exercise. J Alzheimers Dis. 2019;69(3):717-27. doi: 10.3233/JAD-181203. [PubMed: 31006687].

20. Kravchun PG, Kadykova OI, Molotyagin DG. Changes in pentraxin-3 level and its interaction with metabolic indices in patients with coronary artery disease and type 2 diabetes mellitus. Wiad Lek. 2019;72(2):181-5. [PubMed: 30903769].

21. Darwish L, Nguyen MM, Saleem M, Eakin KA, Herrmann N, Sugamori KS, et al. Lower serum osteocalcin concentrations in patients with type 2 diabetes and relationships with vascular risk factors among patients with coronary artery disease. $J$ Diabetes Complications. 2019;33(5):390-7. doi: 10.1016/j. jdiacomp.2019.01.003. [PubMed: 30799280].

22. Ruf J, Vairo D, Paganelli F, Guieu R. Extracellular vesicles with ubiquitinated adenosine A2A receptor in plasma of patients with coronary artery disease. J Cell Mol Med. 2019;23(10):6805-11. doi: 10.1111/jcmm.14564. [PubMed: 31444994].

23. George AK, Majumder A, Ice H, Homme RP, Eyob W, Tyagi SC, et al. Genetics of hyperhomocysteinemia and 1-carbon metabolism: implications for retinal structure and eye functions. Can J Physiol Pharmacol. 2020;98(2):51-60. doi: 10.1139/cjpp-2019-0236. [PubMed: 31369712].

24. Hu Y, Xu Y, Wang G. Homocysteine levels are associated with endothelial function in newly diagnosed type 2 diabetes mellitus patients. Metab Syndr Relat Disord. 2019;17(6):323-7. doi: 10.1089/met.2019.0011. [PubMed: 31045466]. 